\title{
Lieu et temps dans les « inductions » de la Renaissance anglaise
}

Jacques Ramel

\section{OpenEdition}

Journals

Édition électronique

URL : http://journals.openedition.org/shakespeare/524

DOI : 10.4000/shakespeare.524

ISSN : 2271-6424

Éditeur

Société Française Shakespeare

Édition imprimée

Date de publication : 1 novembre 1989

Pagination : 141-150

Référence électronique

Jacques Ramel, «Lieu et temps dans les « inductions » de la Renaissance anglaise », Actes des congrès de la Société française Shakespeare [En ligne], 6| 1989, mis en ligne le 01 janvier 2007, consulté le 06 mai 2019. URL : http://journals.openedition.org/shakespeare/524 ; DOI : 10.4000/ shakespeare.524 


\section{SOCIETE FRANCAISE SHAKESPEARE}

\section{Actes du Congrès - 1984}

\section{LIEU ET TEMPS}

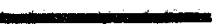

Directeur de la publication Jean FUZIER 


\section{Lieu et Temps dans les Inductions de la Renaissance anglaise.}

Si le prologue de la Seconde Partie d'Henri $I V^{1}$, dit par la Rumeur, est intitulé "Induction" dans l'in-folio, l'usage qui est fait de ce mot actuellement est plus étroit. Synonyme d'introduction, le mot est arrivé à ne plus être utilisé que pour un type très particulier de début de pièce correspondant à des critères bien précis. Pour reprendre la définition donnée par Steven Carter Young, "an induction is a dramatic action which precedes a full-length play. It contains at least two speaking roles, and exists on a different narrative plane from that of the central work $»^{2}$. En appliquant rigoureusement ces critères, nous trouvons, dans les pièces écrites en Angleterre entre 1576 et 1642, cinquante-six exemples d'inductions.

Pour le prologue, le problème du lieu et du temps ne se pose guère. Le personnage du Prologue présente la pièce. Il est un intermédiaire entre les hommes de théâtre et les spectateurs, représentant les premiers auprès des seconds, parlant au nom de l'auteur ou des acteurs. Il est présence physique devant les spectateurs et les paroles qu'il déclame ne peuvent être prononcées à un autre moment, ni ailleurs que là où il se trouve: son rôle n'a de sens que parce qu'il est sur la scène, devant la salle, au moment où va débuter la représentation. 
Cette situation très particulière du Prologue, qui fait de lui l'exact contemporain des spectateurs, lui permet de s'adresser directement à eux à la seconde personne du pluriel, alors qu'il utilisera pour lui-même soit la première personne du singulier, en tant que personnage, soit la première personne du pluriel, en tant que porteparole de la troupe. Ainsi, le Prologue d'Henri $V$ passe-t-il du pluriel, lorsqu'il évoque les difficultés techniques qu'il y a à présenter sur scène une épopée :

... or may we cram

Within this wooden $O$ the very casques

That did affright the air at Agincourt?

au singulier pour parler de lui-même en tant que personnage :

... for the which supply,

Admit me Chorus to this history.

Il existait également une tradition de prologues atypiques, qui au lieu d'être dits par le personnage neutre interchangeable, vêtu de sa longue cape de velours noir, venaient d'un personnage particulier, historique ou allégorique. Ainsi, dans Périclès, Gower, le poète du quatorzième siècle, revient-il présenter la pièce. Disparu depuis longtemps, il apparaît sous la forme d'un fantôme, ce qui lui permet de voyager dans le temps et l'espace pour venir jusque devant les spectateurs contemporains. Le rapport qu'il a avec ces spectateurs est exactement le même que celui du Prologue traditionnel; il se déclare présent sur scène, devant les spectateurs :

From ashes ancient Gower is come...

To glad your ears and please your eyes.

De la même façon, Machiavel introduit Le Juif de Malte tout en respectant les conventions du Prologue. Etant lui aussi un fantôme, il peut venir jusque devant les spectateurs présenter la pièce :

... I come not, I,

To read a lecture here in Britain, But to present the tragedy of a Jew. 
Lorsqu'on aborde les inductions, la question se complique. En effet, tandis que par définition, le Prologue parle devant les spectateurs présents, que le temps et le lieu du texte coïncident exactement avec le temps et le lieu de la représentation, l'induction, elle, est fondée sur l'illusion dramatique, puisqu'elle est jeu théâtral. La recherche de cette illusion implique que l'auteur a dû choisir un lieu, un temps pour l'induction. Nous serons donc amenés à étudier ce choix d'un lieu et d'un temps pour l'induction, à la fois par rapport à la pièce elle-même et par rapport au monde des spectateurs.

Il est évident que les problèmes seront différents selon les différents genres d'inductions. Nous étudierons donc les inductions selon leur répartition traditionnelle en trois grands groupes, que nous appellerons, suivant en cela S.C. Young, extra-dramatiques, narratives et allégoriques ${ }^{3}$.

Les inductions extra-dramatiques ont en commun de présenter des personnages qui semblent appartenir au monde des spectateurs. Ces personnages sont fréquemment l'auteur de la pièce, des acteurs, ou un petit nombre de spectateurs choisis. On reconnaît bien sûr ici le genre d'induction que Jonson a illustré.

De notre point de vue, ces inductions sont assez simples. Suivant en cela les prologues, elles posent en général clairement la coïncidence complète entre temps et lieu du texte et temps et lieu de la représentation. Les personnages de ces inductions reconnaissent être sur une scène, juste avant une représentation. La distance qui les sépare de la pièce elle-même est très grande ; ils sont par contre très proches des spectateurs.

Cette proximité avec le monde des spectateurs peut être renforcée lorsque, comme cela est parfois le cas, un acteur joue son propre rôle dans ce genre d'induction. Dans l'induction d'Antonio and Mellida (Marston, 1599), les acteurs viennent présenter les rôles qu'ils vont jouer dans la pièce. Dans l'induction ajoutée par John Webster à The Malcontent (Marston, 1604), certains acteurs jouent leur propre rôle: Harry Condell joue, sur la scène du Globe, quelques minutes avant le début d'une représentation de The Malcontent, 
le rôle de Harry Condell venant sur la scène du Globe, quelques minutes avant le début d'une représentation de The Malcontent. Il s'agit là d'un cas limite où le théâtre se superpose parfaitement à la réalité. Le seul risque est celui de voir disparaître la notion-même d'illusion théâtrale : avons-nous encore affaire à du théâtre ? Où s'arrête la vie, où commence le théâtre ? C'est sans doute pour affirmer clairement la théâtralité de la situation que l'auteur a pris soin de confier, dans cette induction, à l'acteur William Sly le rôle d'un spectateur qui demande à rencontrer l'acteur William Sly, affirmant ainsi que ce que nous voyons sur scène est bien du théâtre.

Il est arrivé que des spectateurs se laissent abuser par cette parfaite superposition du théâtre et de la réalité, dans ce genre d'induction. On sait que lors d'une représentation de Periander (John Sansbury, 1608) à St John's College, Cambridge, une induction de ce genre faillit mal se terminer pour l'un des acteurs jouant le rôle d'un perturbateur :

Nobody at first took him for an actor. The Chiefest in the Hall commanded that notice should be taken of him, that he might afterwards be punished for his boldness, but as soon as it once appeared that he was an actor their disdain and anger turned to much pleasure and content ${ }^{4}$.

Mais cette recherche de l'illusion théâtrale peut s'exercer dans une direction toute différente. Les inductions-cadres ("framing inductions"), ou inductions narratives, comme les appelle S.P. Olmsted ${ }^{5}$, sont en fait des pièces de théâtre rudimentaires. L'auteur a créé, dans le cadre d'une seule représentation, deux pièces qui ne se différencieront que par leur longueur et leur importance relative. Ainsi, le spectateur de La mégère apprivoisée assiste-t-il d'abord à une courte pièce sur Sly l'ivrogne. Ce pectateur va voir La mégère apprivoisée, et non la pièce sur Sly; et pourtant, La mégère apprivoisée, ou .tout au moins sa représentation, fait bel et bien partie de la pièce sur 
Sly, puisque c'est dans le cadre de cette pièce-ci que celle-là est représentée.

On peut penser que la justification de ce redoublement de la pièce se trouve dans l'articulation qui lie les deux parties entre elles. Mais la nature de cette articulation est souvent difficile à déterminer. Pourquoi avoir fait précéder La mégère apprivoisée de la farce jouée à Sly? Quel lien peut-on voir entre Sly et l'histoire de Katharina? L'auteur de la pièce anonyme Une mégère apprivoisée (1589 ?) tente d'apporter une réponse, puisqu'on voit dans cette pièce Sly reprendre la parole à la fin de la représentation, et déclarer : "I know now how to tame a shrew ". Mais il est peu croyable que la pièce n'existe que pour arriver à cette constatation. Il faut bien convenir que n'importe quelle pièce pourrait être introduite par les scènes avec Sly et que le lien ne semble pas être thématique.

Les premières scènes, qui se déroulent dans la campagne anglaise contemporaine, pourraient-elles servir simplement d'étape sur le chemin qui nous mènera du théâtre londonien à Padoue? Certes, nous n'avons nul besoin de cette étape, et les dramaturges anglais nous ont habitués à des déplacements rapides effectués sans ménagements de Sicile en Bohême ou de Wittenberg à Rome. Mais, d'une façon curieuse, les autres inductions du même type que nous avons conservées se situent toutes entre le monde du spectateur et celui de la pièce qu'elles introduisent, soit dans le temps, soit dans l'espace. Si fournir une étape dans le dépaysement n'était pas le but recherché, en tout cas aucun dramaturge de l'époque n'a osé faire le contraire et nous entraîner plus loin dans le temps ou dans l'espace dans son induction-cadre que dans la pièce elle-même. Ainsi, The Old Wives Tale (Peele, 1590) nous fait transiter par le cadre réaliste d'une chaumière dans la forêt anglaise avant de nous plonger dans le monde merveilleux des contes de fées.

Restent deux pièces que S.C. Young classe parmi les pièces à inductions extra-dramatiques, car celles-ci concernent principalement les conditions de la représentation. Mais cette représentation n'a pas lieu dans le Londres contemporain, contrairement aux autres pièces du même genre, et il semble préférable de les faire entrer dans ce groupe de pièces à inductions-cadres. Dans The Downfall of Robert Earl of Huntington (Chettle et Munday, 1598), les personnages de l'induction, conduits par le poète John Skelton, sont tous des 
membres de la Cour du Roi Henri VIII. Les acteurs sont présents, ils reçoivent des instructions sur leur rôle; les spectateurs eux-mêmes se voient confier un rôle : ils seront les membres de la Cour pour qui la représentation aura lieu. Il y a donc bien là création d'une illusion théâtrale indépendante de celle de la pièce qui suivra et qui nous entraînera, elle, dans l'Angleterre de Robin des Bois. Ici encore, il est difficile de concilier les deux parties de la représentation et de comprendre l'articulation qui les lie. Le spectateur a l'impression qu'on lui fait remonter le temps avec ménagement, en prévoyant en chemin une pause qui n'était guère nécessaire.

L'autre pièce, qui est encore plus curieuse, est Four Plays, or Moral Representations, in One (Fletcher et Field ?, 1612), en fait un ensemble de quatre pièces courtes liées par leur cadre : un mariage royal à la Cour du Portugal entre le Roi Emmanuel I et Isabelle de Castille. Le spectateur assiste en fait à la représentation d'une pièce sur un mariage royal ibérique, au cours duquel sont jouées des scènes de théâtre qui sont situées, pour la première dans la Grèce antique, pour la seconde à Milan, la troisième à Angers, alors que la dernière est allégorique. Le cadre fournit un ancrage dans le temps et dans l'espace auquel est renvoyé le spectateur entre les différents spectacles. Mais nous atteignons ici les limites de la notion de pièce à induction: nous sommes très près du procédé de la pièce dans la pièce. Si La mégère apprivoisée présentée à Sly fait partie d'un tout que nous appelons La mégère apprivoisée, alors que The Murder of Gonzago, joué devant la Cour du Danemark, fait partie d'un tout que nous appelons Hamlet, c'est que nous nous basons sur l'importance relative des deux spectacles proposés dans le cadre de chaque représentation. Dans Four Plays, or Moral Representations, in One, ce qui devrait être la pièce à proprement parler a éclaté en quatre spectacles différents, chacun avec ses personnages, son intrigue, son lieu, son temps ; d'autre part, l'induction a pris de l'importance : ses personnages commentent chaque pièce et ce sont eux qui teminent la représentation, à tel point que nous pouvons hésiter sur le classement de cette pièce. 
Jusqu'ici, nous avons étudié brièvement les inductions extradramatiques, qui appartiennent au temps et à l'espace du monde des spectateurs, et les inductions-cadres auxquelles elles s'opposent et qui, elles, sont des entités théâtrales autonomes, avec un temps et un espace qui leur sont propres. Le troisème groupe d'inductions est plus vague que les deux autres et ne forme pas un ensemble homogène. Young les définit d'ailleurs non du point de vue dramaturgique, mais par la nature des personnages qui y apparaissent : il s'agit d'inductions où se cotoient des fantômes, des personnages de la mythologie grecque ou latine et des allégories.

Le lieu et le temps de ces inductions sont en général ceux des spectateurs. Il n'y a rien de surprenant à cela, puisqu'un recensement rapide des inductions de ce type montre qu'il s'agit souvent de formes étoffées du prologue, dit ici par plusieurs personnages. Un exemple caractéristique montrera le fonctionnement de ce prologue déguisé en induction. Au début de The Valiant Welshman (R.A., 1612), la Fortune réveille un vieux Barde pour lui demander de raconter une histoire. La Fortune ne s'adresse pas aux spectateurs, ne reconnaît pas leur présence et disparaît très vite, laissant seul le Barde, qui récite alors un prologue. De fait, le Barde joue bien le même rôle traditionnel du Prologue que Gower ou Machiavel, après avoir été introduit sur scène par une courte induction.

Aucun des types de personnages de ces inductions ne s'oppose à ce que le temps et le lieu de ces scènes soient ceux du spectateur. Les allégories sont bien sûr des abstractions intemporelles. Les personnages mythologiques sont aussi sensés pouvoir se déplacer dans le temps et dans l'espace à leur guise. Les autres personnages sont bien liés à un lieu et à une époque, mais ils sont maintenant morts et ne sont présentés que sous la forme de fantômes, qui partagent les qualités des allégories et des personnages mythologiques; ils ont pu voyager à travers temps et espace pour venir jusqu'à nous.

Ces fantômes, réveillés pour les inductions, introduisent l'histoire de la pièce qui suit. Ils viennent en général du lieu et du temps de la pièce pour nous y entraîner derrière eux, comme s'ils étaient condamnés à hanter le cadre de leur vie passée. Ainsi Fuimus Troes (Jasper Fisher, 1625), pièce qui se déroule au moment de l'invasion de la Grande-Bretagne par les Romains, est-elle précédée d'une induction comportant deux généraux antiques, l'un britannique et 
l'autre romain; Julia Agrippina Empress of Rome (Thomas May, 1628) est introduite par le fantôme de Caligula; Plutophthalmia Plutogamia (T. Randolph, 1627), adaptation d'Aristophane, est présentée par le fantôme d'Aristophane lui-même.

Ce fantôme qui vient nous chercher et nous entraîne à sa suite peut connaître une variante, celle du fantôme qui surgit du passé pour nous expliquer le présent. Dans l'induction de $A$ Game at Chess (Middleton, 1624), Ignace de Loyola abandonne l'Espagne du début du seizième siècle pour venir introduire une pièce antiespagnole et anti-catholique située dans le monde contemporain des spectateurs. On peut rapprocher ce genre d'induction du prologue du Juif de Malte où le fantôme de Machiavel revient pour expliquer le présent.

La présence du spectateur, que l'on vient ainsi solliciter, est donc presque toujours reconnue, en général ouvertement, parfois de façon plus ironique: dans l'induction de The Conspiracy (Henry Killigrew, 1635), Diane s'étonne qu'on ait joué un tragédie lors du mariage de l'une de ses nymphes. La Tragédie elle-même répond qu'une comédie aurait été un genre trop bas pour une aussi noble assemblée, feignant ainsi de prendre les spectateurs londoniens pour l'assemblée des dieux invités au mariage. Dans Love's Mistress (Thomas Heywood, 1634), l'induction met en scène Apulée, auteur de la fable présentée dans la pièce, et Midas, faux spectateur ignare à qui tout doit être expliqué. Apulée oppose Midas qui ne comprend rien aux spectateurs qui, eux, n'ont besoin d'aucune explication - mais qui profiteront cependant de celles qu'il fournira à Midas :

Though thy vain doubts be most familiar

To those judicious hearers, well experienc'd

As well in matters moral as divine,

To thee I'll make it plain.

En fait, ces inductions allégoriques qui sont bien disparates semblent pouvoir être classées en deux groupes. Dans un premier groupe pourraient être réunies les inductions allégoriques qui sont inutiles à la compréhension de la pièce. Ce sont les innombrables inductions qui ne servent qu'à avertir le spectateur du genre de la pièce qu'il va voir, ou à justifier son auteur, par l'artifice d'une dispute entre la 
Comédie et la Tragédie ou l'Envie ; leur présence n'est pas plus indispensable pour le bon déroulement de la représentation que celle de la plupart des prologues. Mais l'autre groupe serait celui des inductions utiles, voir même indispensables, à la compréhension de la pièce, ou qui, en tout cas, sont liées à la pièce d'une façon quasiment organique. On trouverait dans ce groupe La tragédie espagnole (Kyd, 1587) : la pièce n'est jouée que pour assouvir la vengeance d'Andréa. Il est certes possible de jouer la pièce en supprimant les scènes d'Andréa et de la Vengeance, mais la pièce serait alors amputée, alors que Mucedorus (anonyme, 1590) ne perdrait pas grand chose à être allégée de son introduction. Semblablement, The Rare Triumphs of Love and Fortune (anonyme, 1582) présente des personnages qui ne sont que les jouets des dieux de l'induction : Vénus et la Fortune ont choisi de s'opposer à travers les personnages de cette tragi-comédie, pour démontrer leur puissance. La longueur de l'induction, qui représente un acte entier, et son lien avec le sujet de la pièce, interdisent de représenter celle-ci amputée de celle-là. Lorsqu'existe ce lien organique entre l'induction et la pièce, la distance entre ces deux niveaux d'illusion théâtrale a tendance à diminuer, alors qu'augmente la distance qui sépare l'induction des spectateurs.

Ainsi, alors que dans l'induction de Mucedorus, la Comédie et l'Envie se chamaillent pour savoir qui pourra nous présenter une pièce, reconnaissant ainsi qu'elles se trouvent dans le même temps et le même lieu que nous, la Vengeance va montrer à Andréa une tragédie dont nous ne serons les témoins que par hasard. Mucedorus est jouée pour noter plaisir, La tragédie espagnole pour satisfaire Andréa ; la Comédie et l'Envie sont venues nous rejoindre dans notre théâtre, mais c'est nous qui partons pour rejoindre la Cour d'Espagne où nous attendent déjà les personnages de l'induction. Et lorsqu'Andréa évoque le voyage qui l'a amené là où nous le voyons :

... we were here

I wot not how, in twinkling of an eye,

il parle de son transfert des Champs Elyséens à la Cour du Roi d'Espagne, non à la scène d'un théâtre londonien, alors que dans l'induction de The True Tragedy of Richard III (anonyme, 1591), c'est sur une scène qu'a lieu le rencontre de la Vérité et de la Poésie: 
Poetry: Truth, well met.

Truth: Thanks, Poetry, what makes thou upon a stage?

L'étude de ces inductions allégoriques suivant le critère de leurs liens spatiaux et temporels avec le monde des spectateurs et avec celui de la pièce qu'elles introduisent montre leur extrême diversité. Alors que certaines sont des prologues étoffés, très proches en cela des inductions extra-dramatiques, d'autres créent, malgré la présence de personnages allégoriques ou mythologiques, un niveau d'illusion théâtrale séparé de la pièce, mais plus encore du monde des spectateurs, reproduisant le schéma des inductions-cadres. Mieux, c'est sans doute parmi elles que l'on trouve les meilleurs exemples d'intégration entre la pièce et son cadre. Du point de vue que nous avons adopté pour cette étude, les inductions allégoriques n'existent donc pas en tant que groupe cohérent et devraient être redistribuées dans les deux autres catégories d'inductions qui sont, elles, nettement contrastées.

Jacques RAMEL

\section{NOTES}

1) Le titre des pièces a été laissé en anglais, sauf s'il s'agit de pièces très connues pour lesquelles il existe un titre français accepté. Chaque titre de pièce sera suivi, à sa première mention, de son auteur et de sa date de composition, sauf dans le cas de Shakespeare et de Marlowe. Les dates sont empruntées à Alfred Harbage, Annals of English Drama, 975-1700, 1940, édition révisée par S. Schoenbaum, London, 1964.

2) Steven Carter Young, The Induction Plays of the Tudor and Stuart Drama, Ph. D. Dissertation, Berkeley, 1970, p. 5.

3) Ibid., p. 20.

4) The Christmas Prince, ed. F. S. Boas, Malone Society Reprints, London, 1922 , p. 286, et S.C. Young, op. cit., p. 289.

5) Sterling Pitkin Olmsted, The Development of the Induction in the English Drama, 1582-1642, Ph. D. Dissertation, Yale, 1940. 